\title{
The most metal-rich intervening quasar absorber known ${ }^{\star}$
}

\author{
C. Péroux ${ }^{1}$, V. P. Kulkarni ${ }^{2}$, J. Meiring ${ }^{2}$, R. Ferlet ${ }^{3}$, P. Khare ${ }^{4}$, J. T. Lauroesch $^{5}$, G. Vladilo ${ }^{6}$, and D. G. York ${ }^{7}$ \\ 1 European Southern Observatory, Garching-bei-München, Germany \\ e-mail: cperoux@eso.org \\ 2 Dept. of Physics and Astronomy, Univ. of South Carolina, Columbia, USA \\ 3 Institut d'Astrophysique de Paris, UMR7095 CNRS, Université Pierre \& Marie Curie, France \\ ${ }^{4}$ Dept. of Physics, Utkal University, Bhubaneswar, India \\ 5 Dept. of Physics and Astronomy, Northwerstern University, Evanston, USA \\ ${ }^{6}$ Osservatorio di Trieste, Trieste, Italy \\ 7 Dept. of Astronomy and Astrophysics, Univ. of Chicago, Chicago, USA
}

Received 17 August 2005 / Accepted 3 January 2006

\section{ABSTRACT}

The metallicity in portions of high-redshift galaxies has been successfully measured thanks to the gas observed in absorption in the spectra of quasars, in the Damped Lyman- $\alpha$ systems (DLAs). Surprisingly, the global mean metallicity derived from DLAs is about $1 / 10$ th solar at $0 \lesssim z \lessgtr 4$ leading to the so-called "missing-metals problem". In this paper, we present high-resolution observations of a sub-DLA system at $z_{\text {abs }}=0.716$ with super-solar metallicity toward SDSS J1323-0021. This is the highest metallicity intervening high-H I quasar absorber currently known, and is only the second super-solar such absorber known to date. We provide a detailed study of this unique object from VLT/UVES spectroscopy. We derive $[\mathrm{Zn} / \mathrm{H}]=+0.61,[\mathrm{Fe} / \mathrm{H}]=-0.51,[\mathrm{Cr} / \mathrm{H}]=<-0.53,[\mathrm{Mn} / \mathrm{H}]=-0.37$, and $[\mathrm{Ti} / \mathrm{H}]=-0.61$. Observations and photoionisation models using the CLOUDY software confirm that the gas in this sub-DLA is predominantly neutral and that the abundance pattern is probably significantly different from a Solar pattern. Fe/Zn and Ti/Zn vary among the main velocity components by factors of $\sim 3$ and $\sim 35$, respectively, indicating non-uniform dust depletion. $\mathrm{Mn} / \mathrm{Fe}$ is super-solar in almost all components, and varies by a factor of $\sim 3$ among the dominant components. It would be interesting to observe more sub-DLA systems and determine whether they might contribute significantly toward the cosmic budget of metals.

Key words. galaxies: abundances - intergalactic medium - quasars: absorption lines - quasars: individual: SDSS J1323-0021

\section{Introduction}

Damped Lyman- $\alpha$ systems (DLAs) seen in absorption in the spectra of background quasars are selected over all redshifts independent of the intrinsic luminosities of the underlying galaxies. They have hydrogen column densities, $\log N_{\mathrm{HI}} \gtrsim 20.3$ and are the major contributors to the neutral gas in the Universe at high redshifts (Storrie-Lombardi \& Wolfe 2000; Péroux et al. $2003 \mathrm{~b}$ ). But it has been suggested that at least some of the $\mathrm{H}$ I lies in systems with $\mathrm{H}$ I column density below that required by the traditional DLA definition, in the "sub-Damped Lyman- $\alpha$ Systems (sub-DLAs)" with $19.0<\log N_{\mathrm{HI}}<20.3$. The DLAs and sub-DLAs offer direct probes of element abundances over $>90 \%$ of the age of the Universe. $\mathrm{Zn}$ is a good probe of the total (gas and solid phase) metallicity, in DLAs because $\mathrm{Zn}$ tracks $\mathrm{Fe}$ in most Galactic stars with $[\mathrm{Fe} / \mathrm{H}]>-3$, it is undepleted on interstellar dust grains, and the lines of the

\footnotetext{
* Based on the UVES observations collected during the DDT ESO prog. ID No. 274.A-5030 at the VLT/Kueyen telescope, Paranal, Chile.
}

dominant ionisation species Zn II are often unsaturated (e.g., Pettini et al. 1999). Abundances of depleted elements such as $\mathrm{Cr}$ or $\mathrm{Fe}$ relative to $\mathrm{Zn}$ probe the dust content and the relative abundances can also yield information about the nucleosynthetic processes (e.g., Pettini et al. 1997; Kulkarni et al. 1997; Péroux et al. 2002; Khare et al. 2004). A study of the cosmological evolution of the $\mathrm{H}$ I column density-weighted mean metallicity in DLAs (e.g., Kulkarni \& Fall 2002) shows surprising results. Contrary to most models of cosmic chemical evolution (e.g., Malaney \& Chaboyer 1996; Pei et al. 1999), recent observations indicate at most a mild evolution in DLA global metallicity with redshift for $0 \lesssim z \lesssim 4$ (Prochaska et al. 2003; Khare et al. 2004; Kulkarni et al. 2005, and references therein). Even theoretical models such as Smoothed-particle-hydrodynamics simulations (Nagamine et al. 2004) predict that the true DLA metallicities could be $1 / 3$ solar at $z=2.5$ and higher at lower redshifts.

Even at $z=2.5$, making a census of the predicted and observed neutral comoving densities of gas, $\Omega$, and metals, $\Omega_{\mathrm{Z}}$, one finds that most of the baryons are in the Lyman- $\alpha$ forest 
but its metal content is extremely low. The measured value of $\Omega_{\mathrm{HI}}$ (DLA) is only a small fraction of $\Omega_{\text {baryons }}$ and the DLA global mean metallicity is about $1 / 10$ th solar. The metallicity of Lyman break galaxies is still poorly constrained; but, in any case, these objects are known to be star-forming galaxies and may not be representative of the normal galaxy population. In total, these three components account for no more than $\approx 10-15 \%$ of what we expect to have been produced by $z=2.5$ (Pettini et al. 2003; Bouché et al. 2005). The missing metals problem in low-redshift DLAs is even more surprising since the high global star formation rate estimates at $z>1.5$ (e.g. Madau et al. 1998) imply that higher metallicities should be expected at low redshift.

It is possible that $\Omega_{Z}$ (DLA) has been underestimated. Metal-rich DLAs could obscure quasars due to their possible high dust content (Fall \& Pei 1993). This may be the reason for the apparent low metallicity in the DLAs observed in optically selected quasars (e.g., Fall \& Pei 1993; Boissé et al. 1998). Recently Vladilo \& Péroux (2005) have shown that the fraction of high-redshift DLAs missed due to dust obscuration, could be up to $50 \%$, which is consistent with the results of surveys of radio selected quasars (Ellison et al. 2001). They have estimated that at $z \sim 2.3$, the real mean metallicity of DLAs could be 5 to 6 times higher than what is observed, which may help alleviate the missing metals problems. Indeed, systems at lower redshift may have significantly more dust at any given metallicity simply because the dust in these objects has had more time to process the elements.

On the other hand, new lines of evidence are pointing toward lower $N_{\mathrm{HI}}$ quasar absorbers like Lyman Limit Systems (LLS) and sub-DLAs being more metal-rich (Péroux et al. 2003a; Jenkins et al. 2005). The dust bias, if real, is also likely to be less severe for metal-rich sub-DLAs as compared to the metal-rich DLAs due to the lower gas and therefore dust content in the former, for a constant dust to gas ratio. Thus, the obscuration bias will affect the DLAs at a lower dust-to-gas ratio as compared to the sub-DLAs. This scenario is consistent with the recent radio surveys (e.g., Vladilo \& Péroux 2005), but still needs to be further quantified observationally. Indeed, supersolar metallicities have been reported at $\log N(\mathrm{H} \mathrm{I})<16$ (e.g., Charlton et al. 2003; Ding et al. 2003; Masiero et al. 2005), but these are more model-dependent owing to ionisation corrections. $\mathrm{Zn}$ measurements exist for only two sub-DLAs at low $z$ : the marginally super-solar sub-DLA toward Q0058+019 with $z_{\mathrm{abs}}=0.61, \log N_{\mathrm{HI}}=20.08$, and $[\mathrm{Zn} / \mathrm{H}]=+0.08$ (Pettini et al. 2000), and the supersolar sub-DLA toward SDSS J1323-0021 with $z_{\mathrm{abs}}=0.72, \log N_{\mathrm{HI}}=20.21$, and $[\mathrm{Zn} / \mathrm{H}]=+0.40$ (Khare et al. 2004). Although Khare et al. reported $[\mathrm{Zn} / \mathrm{H}]=+0.40$ for this latter absorber, the modest resolution of their MMT data could not resolve the Mg I+Zn II $\lambda 2026$ and Cr II+Zn II $\lambda 2062$ blends. The extent of line saturation on the derived column densities was also unclear. With the goal of addressing these issues with high-resolution data, we obtained VLT/UVES spectra of this quasar, which are presented here. These new data are essential to confidently determine the metallicity by minimising the problem of line saturation. Section 2 presents the observational set-up and data reduction process, while Sect. 3 presents the analysis. Section 4 provides a discussion of the results.

\section{Observations and data reduction}

Spectra of SDSS J1323-0021 $\left(z_{\mathrm{em}}=1.390\right.$; SDSS mag $g=18.49)$ were acquired in service mode as Director's Discretionary Time (DDT) on 3rd of March and 13th of March 2005 with the high-resolution UVES spectrograph mounted on Kueyen Unit 2 VLT (D'Odorico et al. 2000). Three exposures of length $4100 \mathrm{~s}, 3500 \mathrm{~s}$ and $4100 \mathrm{~s}$ were obtained with standard $390+562$ settings thus providing a wavelength coverage of 3300 $\mathrm{A}-4400 \AA, 4700 \AA-5600 \AA$ and $5800 \AA-6600 \AA$.

The data were reduced using the most recent version of the UVES pipeline to accommodate for the new format of the raw fits file (version: uves/2.1.0 flmidas/1.1.0). Master bias and flat images were constructed using calibration frames taken the closest in time to the science frames. The science frames were extracted with the "optimal" option. The spectrum was then corrected to vacuum heliocentric reference. The resulting spectra were combined weighting each spectrum with its signalto-noise. The final spectra have resolution of $4.7 \mathrm{~km} \mathrm{~s}^{-1}$ at Zn II 22026 . The spectra were divided into $100 \AA$ regions, and each region normalised using cubic spline functions of orders 1 to 5 .

\section{Analysis}

Several lines of $\mathrm{Zn}$ II, Cr II, Fe II, Mn II, and Ti II were detected at $z_{\mathrm{abs}}=0.716$. Fe I, Zn I, and Co II were not detected. The column densities were estimated by fitting multi-component Voigt profiles to the observed absorption lines using the program FITS6P (Welty et al. 1991) that evolved from the code used by Vidal-Madjar et al. (1977). FITS6P minimizes the $\chi^{2}$ between the data and the theoretical Voigt profiles convolved with the instrumental profile. The atomic data were adopted from Morton (2003).

The absorption profiles show a complex velocity structure with a total of 16 components needed. The velocity and Doppler b parameters of the various components were estimated from the Mg I, Mg II, and Fe II lines. The component at $12 \mathrm{~km} \mathrm{~s}^{-1}$ is negligible in most species except $\mathrm{Mg} \mathrm{I}, \mathrm{Zn}$ II and $\mathrm{Cr}$ II. The component at $194 \mathrm{~km} \mathrm{~s}^{-1}$ is negligible in all species but $\mathrm{Mg}$ II. The component at $-121 \mathrm{~km} \mathrm{~s}^{-1}$ is detected in $\mathrm{Mg}$ II, Fe II, and Mn II, but not in Mg I, Zn II, Cr II, or Ti II. The best-fit column densities in the individual components and their uncertainties were estimated assuming the same fixed $b$ and $v$ values for all species in a given component (Fig. 1). The results of the profile fitting analysis are summarised in Table 1. Column densities in the few weak components that could not be well-constrained due to noise are marked with "..."; their contributions to the total column densities (listed in Table 2) are negligible. As an additional check, we also estimated the total column densities using the apparent optical depth (AOD; Savage \& Sembach 1991) method for the various detected lines and obtained results consistent with those from the profile-fitting method. The agreement was within $0.01 \mathrm{dex}$ for $\mathrm{Mg} \mathrm{I}$ and $\mathrm{Mn} \mathrm{II}$, within 0.1 dex for Fe II and Zn II, and within 0.15 dex for Ti II.

The Mg I $\lambda 2026.5$ contribution to the $\mathrm{Zn}$ II $\lambda 2026.1$ line was estimated using the component parameters for $\mathrm{Mg}$ I derived from the $\mathrm{Mg}$ I $\lambda 2852$ profile. This contribution, 

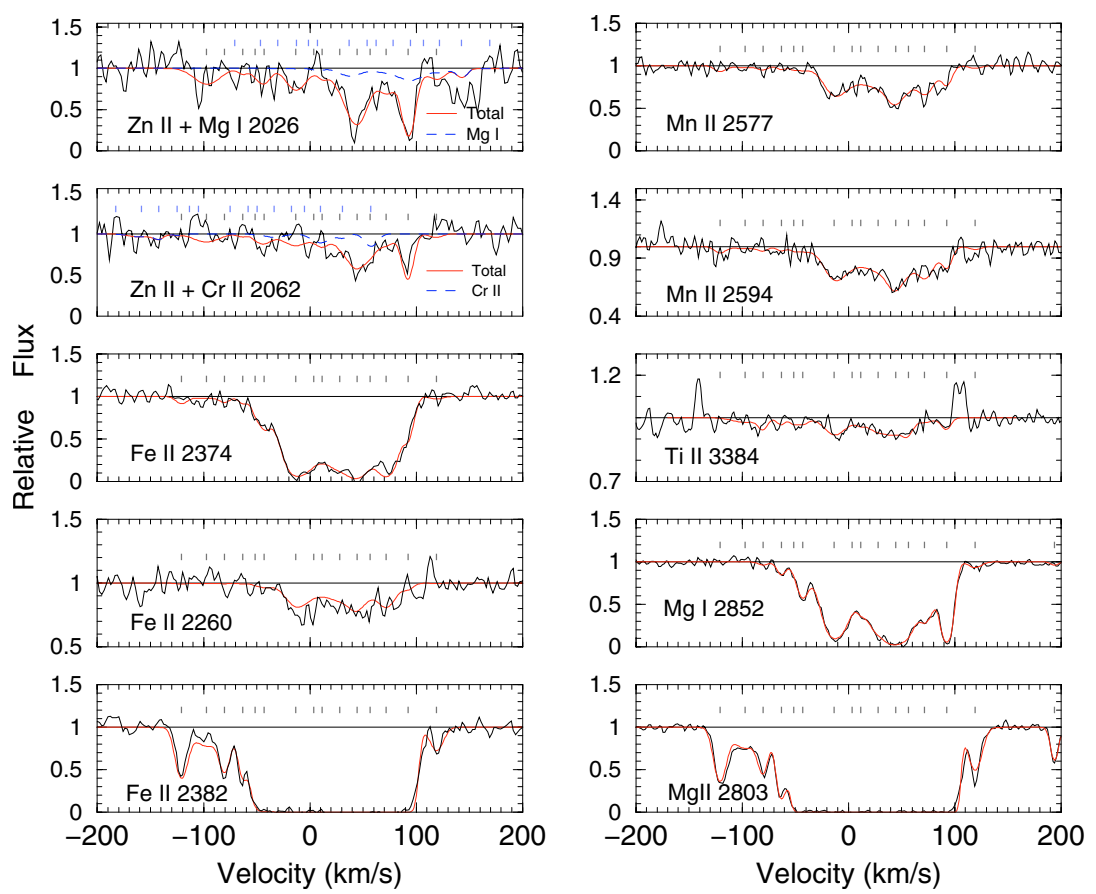

Fig. 1. Velocity plots of some of the element lines detected at $z_{\mathrm{abs}}=0.716$ toward SDSS J1323-0021. The black tick-marks indicate positions of the components and the red curves denote the best-fit profiles. In the two top left panels, the upper set of blue tickmarks indicate positions of the components of Mg I $\lambda 2026$ and Cr II $\lambda 2062$, respectively, whilst the blue curves show their contributions to the absorption profiles. Note that different ordinates are used on the sub-frames.

indicated by a dashed blue curve in the top left panel of Fig. 1, was a small fraction of the observed strength of the $\lambda 2026$ line. The remaining part of the $\lambda 2026$ line was fitted with a 15-component model for Zn II, using the same $b$ values and velocities, but varying the column densities in the individual components. The $\mathrm{Zn}$ II fit thus obtained was used to estimate the $\mathrm{Zn}$ II contribution to the $\lambda 2062$ line. The remaining part of the $\lambda 2062$ line was fitted with a 15 -component model for Cr II, using the same set of $b$ values and velocities. This was the only available estimate for $\mathrm{Cr}$ II, since the $\mathrm{Cr}$ II $\lambda \lambda 2056$, 2066 lines lie in noisy regions and are undetected. The relatively large errors in the Cr II column densities arise from the noisy nature of the $\lambda 2062$ line. The Fe II column densities in the components at low velocities were constrained by using the Fe II $\lambda \lambda 2260,2374$ lines, since the stronger Fe II lines are saturated. The weaker Fe II components at high positive and negative velocities were constrained in column density using the $\lambda \lambda 2374,2382,2600$ lines, since these components are poorly constrained by the weaker $\lambda \lambda 2260,2374$ lines. The Mg II $\lambda 2796,2803$ profiles were fitted together, but provide only a lower limit to $\mathrm{Mg}$ II owing to saturation in the central components. The relative abundances were calculated using solar abundances from Asplund et al. (2005), adopting the mean of photospheric and meteoritic values for $\mathrm{Mg}, \mathrm{Ti}, \mathrm{Cr}$, $\mathrm{Fe}, \mathrm{Zn}$, and the meteoritic value for $\mathrm{Mn}$.

One concern is that sub-DLAs may be partially ionised in $\mathrm{H}$, artificially enhancing the ratio of $\mathrm{Zn}$ II to $\mathrm{H}$ I, for instance. To investigate the ionisation corrections, we used the CLOUDY software package (version 94.00, Ferland 1997) and computed photoionisation models assuming ionisation equilibrium and a solar abundance pattern. We thus obtained the theoretical column density predictions for any ionisation state of all observed ions as a function of the ionisation parameter $U$. Our findings confirm the observations: from a comparison of the observed and theoretical $\mathrm{Mg} \mathrm{II} / \mathrm{Mg}$ I ratios, we deduced that the gas in this sub-DLA is predominantly neutral $(\log U<-5)$ and the overall abundance pattern is probably not solar. This latter point is also clear from the relative abundances listed for each component in Table 1. It should be pointed out that there are no third ionisation stage detected in the system under study. Nevertheless, we do have Ti II, which has the same ionisation potential as $\mathrm{H} \mathrm{I}$. The fact that $\mathrm{Ti}$ is not suppressed compared to Fe or Mn also implies that the gas is neutral with low ionisation parameter.

\section{Discussion and conclusions}

Table 2 lists the abundances, using $\log N_{\mathrm{HI}}=20.21_{-0.18}^{+0.21}$ (Khare et al. 2004) that we derived from Voigt profile fitting of the damped Ly- $\alpha$ line in the publicly available HST/STIS spectrum of SDSS J1323-0021 (program GO 9382; PI: Rao). Rao et al. (2006) obtained $\log N_{\mathrm{HI}}=20.54_{-0.15}^{+0.16}$ from the same data set. We use the former value since it gives a smaller residual with respect to the data and therefore regard this absorber as a sub-DLA. For either $N_{\mathrm{HI}}$, the strength of the $\mathrm{Zn}$ II lines detected in our UVES spectrum implies a super-solar metallicity. Using the standard definition: $[X / \mathrm{H}]=\log [N(X) / N(\mathrm{H})]_{\text {DLA }}-$ $\log [N(X) / N(\mathrm{H})]_{\odot}$, we find $[\mathrm{Zn} / \mathrm{H}]=+0.61$.

In principle, if $\mathrm{Mg}$ I $\lambda 2852$ were substantially saturated, the contribution of $\mathrm{Mg}$ I $\lambda 2026$ could be higher than our best-fit estimate. However, based on our apparent optical depth measurements and profile-fitting results, we estimate that it would 
Table 1. Parameter fit to the $z_{\text {abs }}=0.716$ sub-DLA model. Velocities and $b$ are in $\mathrm{km} \mathrm{s}^{-1}$ and N's are in $\mathrm{cm}^{-2}$.

\begin{tabular}{|c|c|c|c|c|c|c|c|c|c|c|c|}
\hline & Vel & $b$ & $\mathrm{Mg} \mathrm{I}$ & Mg II & Fe II & Zn II & Cr II & Mn II & Ti II & {$[\mathrm{Fe} / \mathrm{Zn}]$} & {$[\mathrm{Mn} / \mathrm{Fe}]$} \\
\hline$N(X)$ & -120.8 & 7.1 & $\ldots$ & $5.63 \mathrm{e} 12$ & $5.82 \mathrm{e} 12$ & $\ldots$ & $\ldots$ & $3.75 \mathrm{e} 11$ & $\ldots$ & $\ldots$ & 0.79 \\
\hline$\sigma$ & $\ldots$ & $\ldots$ & $\ldots$ & $1.66 \mathrm{e} 11$ & $4.08 \mathrm{e} 11$ & $\ldots$ & $\ldots$ & $1.26 \mathrm{e} 11$ & $\ldots$ & $\ldots$ & $\ldots$ \\
\hline$N(X)$ & -96.9 & 16.3 & $\ldots$ & $3.65 \mathrm{e} 12$ & $3.64 \mathrm{e} 12$ & $2.32 \mathrm{e} 12$ & $<2.81 \mathrm{e} 12$ & $2.97 \mathrm{e} 11$ & $2.09 \mathrm{e} 11$ & -2.65 & 0.89 \\
\hline$\sigma$ & $\ldots$ & $\ldots$ & $\ldots$ & $1.21 \mathrm{e} 11$ & $4.31 \mathrm{e} 11$ & $6.53 \mathrm{e} 11$ & $\ldots$ & $1.93 \mathrm{e} 11$ & $1.27 \mathrm{e} 11$ & $\ldots$ & $\ldots$ \\
\hline$N(X)$ & -80.1 & 6.2 & $3.16 \mathrm{e} 10$ & $3.55 \mathrm{e} 12$ & $3.92 \mathrm{e} 12$ & $\ldots$ & $<1.69 \mathrm{e} 12$ & $2.09 \mathrm{e} 11$ & $1.86 \mathrm{e} 11$ & $\ldots$ & 0.71 \\
\hline$\sigma$ & $\ldots$ & $\ldots$ & $1.15 \mathrm{e} 10$ & $1.36 \mathrm{e} 11$ & $3.69 \mathrm{e} 11$ & $\ldots$ & $\ldots$ & $1.23 \mathrm{e} 11$ & $8.41 \mathrm{e} 10$ & $\ldots$ & $\ldots$ \\
\hline$N(X)$ & -62.9 & 5.5 & $1.20 \mathrm{e} 11$ & $8.13 \mathrm{e} 12$ & $4.25 \mathrm{e} 12$ & $<3.10 \mathrm{e} 11$ & $<4.77 \mathrm{e} 11$ & $2.01 \mathrm{e} 11$ & $1.07 \mathrm{e} 11$ & -1.71 & 0.65 \\
\hline$\sigma$ & $\ldots$ & $\ldots$ & $1.23 \mathrm{e} 10$ & $3.68 \mathrm{e} 11$ & $4.22 \mathrm{e} 11$ & $\ldots$ & $\ldots$ & $1.01 \mathrm{e} 11$ & $8.00 \mathrm{e} 10$ & $\ldots$ & $\ldots$ \\
\hline$N(X)$ & -51.4 & 5.0 & $<1.33 \mathrm{e} 10$ & $>2.86 \mathrm{e} 12$ & $5.74 \mathrm{e} 12$ & $<2.56 \mathrm{e} 11$ & $\ldots$ & $1.44 \mathrm{e} 11$ & $<6.92 \mathrm{e} 10$ & -1.49 & 0.38 \\
\hline$\sigma$ & $\ldots$ & $\ldots$ & $\ldots$ & $\ldots$ & $1.30 \mathrm{e} 12$ & $\ldots$ & $\ldots$ & $1.19 \mathrm{e} 11$ & $\ldots$ & $\ldots$ & $\ldots$ \\
\hline$N(X)$ & -43.0 & 7.4 & $4.80 \mathrm{e} 11$ & $>6.01 \mathrm{e} 13$ & $2.41 \mathrm{e} 13$ & $1.07 \mathrm{e} 12$ & $\ldots$ & $1.98 \mathrm{e} 11$ & $1.32 \mathrm{e} 11$ & -1.49 & -0.11 \\
\hline$\sigma$ & $\ldots$ & $\ldots$ & $2.29 \mathrm{e} 10$ & $\ldots$ & $3.54 \mathrm{e} 12$ & $5.18 \mathrm{e} 11$ & $\ldots$ & $1.43 \mathrm{e} 11$ & $9.85 \mathrm{e} 10$ & $\ldots$ & $\ldots$ \\
\hline$N(X)$ & -13.0 & 12.5 & $3.04 \mathrm{e} 12$ & $>2.38 \mathrm{e} 14$ & $1.97 \mathrm{e} 14$ & $2.57 \mathrm{e} 12$ & $\ldots$ & $2.40 \mathrm{e} 12$ & $4.79 \mathrm{e} 11$ & -0.96 & 0.07 \\
\hline$\sigma$ & $\ldots$ & $\ldots$ & $1.48 \mathrm{e} 11$ & $\ldots$ & $2.54 \mathrm{e} 13$ & $6.22 \mathrm{e} 11$ & $\ldots$ & $3.14 \mathrm{e} 11$ & $2.16 \mathrm{e} 11$ & $\ldots$ & $\ldots$ \\
\hline$N(X)$ & 3.9 & 30.5 & $2.36 \mathrm{e} 12$ & $>1.48 \mathrm{e} 14$ & $4.00 \mathrm{e} 14$ & $\ldots$ & $\ldots$ & $5.33 \mathrm{e} 12$ & $<4.17 \mathrm{e} 11$ & $\ldots$ & 0.10 \\
\hline$\sigma$ & $\ldots$ & $\ldots$ & $2.03 \mathrm{e} 11$ & $\ldots$ & $2.63 \mathrm{e} 13$ & $\ldots$ & $\ldots$ & $5.28 \mathrm{e} 11$ & $\ldots$ & $\ldots$ & $\ldots$ \\
\hline$N(X)$ & 12.0 & 5.0 & $1.44 \mathrm{e} 11$ & $\ldots$ & $\ldots$ & $<3.66 \mathrm{e} 11$ & $<3.20 \mathrm{e} 11$ & $\ldots$ & $<3.89 \mathrm{e} 10$ & $\ldots$ & 0.01 \\
\hline$\sigma$ & $\ldots$ & $\ldots$ & $4.81 \mathrm{e} 10$ & $\ldots$ & $\ldots$ & $\ldots$ & $\ldots$ & $\ldots$ & $\ldots$ & $\ldots$ & $\ldots$ \\
\hline$N(X)$ & 28.3 & 12.0 & $2.06 \mathrm{e} 12$ & $>7.14 \mathrm{e} 13$ & $1.15 \mathrm{e} 14$ & $1.53 \mathrm{e} 12$ & $<2.37 \mathrm{e} 12$ & $1.28 \mathrm{e} 12$ & $3.80 \mathrm{e} 11$ & -0.97 & 0.03 \\
\hline$\sigma$ & $\ldots$ & $\ldots$ & $1.25 \mathrm{e} 11$ & $\ldots$ & $2.94 \mathrm{e} 13$ & $7.60 \mathrm{e} 11$ & $\ldots$ & $2.86 \mathrm{e} 11$ & $1.53 \mathrm{e} 11$ & $\ldots$ & $\ldots$ \\
\hline$N(X)$ & 44.5 & 11.0 & $4.76 \mathrm{e} 12$ & $>2.30 \mathrm{e} 13$ & $2.89 \mathrm{e} 14$ & $7.76 \mathrm{e} 12$ & $\ldots$ & $4.34 \mathrm{e} 12$ & $4.37 \mathrm{e} 11$ & -1.27 & 0.16 \\
\hline$\sigma$ & $\ldots$ & $\ldots$ & $4.37 \mathrm{e} 11$ & $\ldots$ & $5.42 \mathrm{e} 13$ & $1.48 \mathrm{e} 12$ & $\ldots$ & $2.90 \mathrm{e} 11$ & $1.22 \mathrm{e} 11$ & $\ldots$ & $\ldots$ \\
\hline$N(X)$ & 56.6 & 6.9 & $8.70 \mathrm{e} 11$ & $>4.62 \mathrm{e} 13$ & $2.83 \mathrm{e} 13$ & $<2.73 \mathrm{e} 11$ & $\ldots$ & $6.30 \mathrm{e} 11$ & $2.69 \mathrm{e} 11$ & -0.83 & 0.33 \\
\hline$\sigma$ & $\ldots$ & $\ldots$ & $1.15 \mathrm{e} 11$ & $\ldots$ & $1.82 \mathrm{e} 13$ & $\ldots$ & $\ldots$ & $1.97 \mathrm{e} 11$ & $9.48 \mathrm{e} 10$ & $\ldots$ & $\ldots$ \\
\hline$N(X)$ & 71.8 & 11.9 & $1.93 \mathrm{e} 12$ & $>5.09 \mathrm{e} 14$ & $3.12 \mathrm{e} 14$ & $2.49 \mathrm{e} 12$ & $6.07 \mathrm{e} 12$ & $3.62 \mathrm{e} 12$ & $1.58 \mathrm{e} 11$ & -0.75 & 0.04 \\
\hline$\sigma$ & $\ldots$ & $\ldots$ & $5.19 \mathrm{e} 10$ & $\ldots$ & $4.29 \mathrm{e} 13$ & $7.30 \mathrm{e} 11$ & $3.24 \mathrm{e} 12$ & $2.22 \mathrm{e} 11$ & $1.05 \mathrm{e} 11$ & $\ldots$ & $\ldots$ \\
\hline$N(X)$ & 92.7 & 6.4 & $2.42 \mathrm{e} 12$ & $>2.99 \mathrm{e} 14$ & $3.76 \mathrm{e} 13$ & $7.35 \mathrm{e} 12$ & $<1.54 \mathrm{e} 12$ & $1.20 \mathrm{e} 12$ & $2.09 \mathrm{e} 11$ & -2.14 & 0.48 \\
\hline$\sigma$ & $\ldots$ & $\ldots$ & $8.51 \mathrm{e} 10$ & $\ldots$ & $4.70 \mathrm{e} 12$ & $1.49 \mathrm{e} 12$ & $\ldots$ & $1.40 \mathrm{e} 11$ & $7.87 \mathrm{e} 10$ & $\ldots$ & $\ldots$ \\
\hline$N(X)$ & 119.3 & 8.0 & $7.45 \mathrm{e} 10$ & $4.49 \mathrm{e} 12$ & $2.33 \mathrm{e} 12$ & $5.13 \mathrm{e} 11$ & $6.01 \mathrm{e} 12$ & $1.98 \mathrm{e} 11$ & $1.02 \mathrm{e} 08$ & -2.19 & 0.91 \\
\hline$\sigma$ & $\ldots$ & $\ldots$ & $1.32 \mathrm{e} 10$ & $4.90 \mathrm{e} 07$ & $2.85 \mathrm{e} 11$ & $4.48 \mathrm{e} 11$ & $3.11 \mathrm{e} 12$ & $1.27 \mathrm{e} 11$ & $1.42 \mathrm{e} 07$ & $\ldots$ & $\ldots$ \\
\hline$N(X)$ & 194.1 & 4.6 & $2.44 \mathrm{e} 10$ & $1.91 \mathrm{e} 12$ & $\ldots$ & $\ldots$ & $\ldots$ & $\ldots$ & $\ldots$ & $\ldots$ & $\ldots$ \\
\hline$\sigma$ & $\ldots$ & $\ldots$ & $1.00 \mathrm{e} 10$ & $7.56 \mathrm{e} 10$ & $\ldots$ & $\ldots$ & $\ldots$ & $\ldots$ & $\ldots$ & $\ldots$ & $\ldots$ \\
\hline
\end{tabular}

take $\sim 8$ times more total $\mathrm{Mg}$ I than the value derived from the $\lambda 2852$ line to contribute the entirely of the $\lambda 2026$ line. Such a high value of $\mathrm{Mg}$ I can be ruled out by the observed profile of the $\lambda 2852$ line. (Of course, such a scenario would also be inconsistent with the observed strength and profile of the $\lambda 2062$ line.) To understand this issue in more detail, we estimated the maximum $\mathrm{Mg}$ I in the dominant components that would still give the shape of the Mg I $\lambda 2852$ profile consistent with the observed profile within the noise level in the continuum. This maximum total $\mathrm{Mg} \mathrm{I}=2.7 e 13$ is about $50 \%$ larger than the best-fit value listed in Table 2. Putting this maximum $\mathrm{Mg} \mathrm{I}$ model in the $\lambda 2026$ line, the corresponding total $\mathrm{Zn}$ II needed to fit the remaining part of the $\lambda 2026$ line would be $2.5 \mathrm{e} 13$, lower by $<10 \%$ from our best-fit value. Thus $[\mathrm{Zn} / \mathrm{H}]$ is at least $>+0.59$, indicating that our result would not be affected much by saturation of $\mathrm{Mg} \mathrm{I} \lambda 2852$. Finally, as an additional check, we also estimated the maximum contribution of Cr II 2062 by rebinning our spectrum by factors of 10 or 20 , measuring the upper limit for $\mathrm{Cr}$ II $\lambda 2056$ in the rebinned spectrum. We then spread this upper limit for N(Cr II) over the 2062 profile, using the velocity model derived from the combination of the lines. We assume the same $\mathrm{Fe} / \mathrm{Cr}$ ratio in all components, taking the percentage of $\mathrm{Cr}$ II in each components relative to total $\mathrm{N}(\mathrm{Cr}$ II) summed over all components to be the same as the corresponding percentage of $\mathrm{Fe}$ II in that component. Fitting the remaining part of the $\lambda 2062$ line with a 15-component model of $\mathrm{Zn}$ II, we estimated $N_{\mathrm{ZnII}}>2.05 e 13$, i.e. $[\mathrm{Zn} / \mathrm{H}]>0.50$.

The abundances of $\mathrm{Fe}, \mathrm{Cr}, \mathrm{Mn}$, and $\mathrm{Ti}$ lie in the range of -0.4 to $-0.6 \mathrm{dex}$, and indicate that this absorber is not only metal-rich, but also very dusty. Using the model from Vladilo (2004), we find that $95 \%$ of the $\mathrm{Fe}$ is in dust phase and the total metallicity is even slightly higher than 0.6 dex. This sightline also shows substantial reddening compared to the SDSS quasar composite $(\Delta(g-i)=0.47$; Khare et al. 2004). Considering only the better-determined components between -13 and $100 \mathrm{~km} \mathrm{~s}^{-1}, \mathrm{Fe} / \mathrm{Zn}$ varies by a factor of $\sim 3$ and $\mathrm{Ti} / \mathrm{Zn}$ varies by $\sim 35$. [Mn/Fe] varies by $\sim 3$, but indicates a super-solar $\mathrm{Mn}$ abundance with respect to $\mathrm{Fe}$ in all components. The relative abundance of $\mathrm{Mn}$ with respect to $\mathrm{Fe}$ is not expected to exceed the solar value as $\mathrm{Mn}$, unlike $\mathrm{Fe}$, has an 
Table 2. Summary of total abundances.

\begin{tabular}{lclc}
\hline \hline Id & $\log N_{\text {total }}$ & $A(X / \mathrm{N})_{\odot}$ & {$[X / \mathrm{H}]^{*}$} \\
\hline $\mathrm{Mg}_{\text {I }}$ & $13.26 \pm 0.01$ & $\ldots$ & $\ldots$ \\
$\mathrm{Mg}_{\text {II }}$ & $>15.15$ & -4.47 & $>-0.58$ \\
Fe II & $15.15 \pm 0.03$ & -4.55 & $-0.51 \pm 0.20$ \\
Zn II & $13.43 \pm 0.05$ & -7.40 & $+0.61 \pm 0.20$ \\
$\mathrm{Cr}_{\text {II }}$ & $<13.33$ & -6.37 & $<-0.52$ \\
$\mathrm{Mn}_{\text {II }}$ & $13.31 \pm 0.02$ & -6.53 & $-0.37 \pm 0.20$ \\
Ti II & $12.49 \pm 0.11$ & -7.11 & $-0.61 \pm 0.22$ \\
\hline
\end{tabular}

* The error bars on $[X / \mathrm{H}]$ include the errors in $\log N(X)$ and $\log N_{\mathrm{HI}}$.

odd atomic number. However, $[\mathrm{Mn} / \mathrm{Fe}]>0$ is often seen in the Galactic interstellar gas due to the stronger depletion of Fe on to dust grains.

To summarise, our high resolution VLT/UVES data have allowed us to alleviate the saturation issue in $\mathrm{Zn}$ II and $\mathrm{Cr}$ II lines and therefore unambiguously prove the supersolar metallicity of the sub-DLA at $z_{\mathrm{abs}}=0.716$ toward SDSS J1323-0021. If the dust obscuration bias for DLAs is indeed significant, as proposed by Vladilo \& Péroux (2005), sub-DLA systems such as the one reported here could be better probes of dusty regions with significant past star formation (e.g. Lauroesch et al. 1996; York et al. 2006), as similar DLA systems will be missed due to dust obscuration. On the other hand, it is also, possible to envisage a scenario where the dust obscuration is not very significant in quasar absorbers (as is indicated by the rising spectrum of gamma-ray burst afterglows). In this scenario it is possible that the sub-DLA systems may indeed be more metal-rich as compared to DLAs as indicated by observations of Péroux et al. (2003a) and by the observations of super-solar metallicity in such systems presented here and in Pettini et al. (2003). With the large-scale spectroscopic surveys of quasars currently underway (e.g. the SDSS, York et al. 2000, 2001), such metal-rich sub-DLA systems may be found in large numbers. If future observations indeed find such systems, sub-DLAs may contribute significantly to the overall global metallicity.

Acknowledgements. We are grateful to ESO director, Catherine Cesarsky, for time allocation to this DDT program, and to the VLT staff for carrying out our observations in service mode. V.P.K. and J.M. acknowledge support from the US National Science Foundation grant AST-0206197.

\section{References}

Asplund, M., Grevesse, N., \& Sauval, A. J. 2005, ASP Conf. Ser., ed. Bash, \& Barnes, 336, 25
Bouché, N., Lehnert, M. D., \& Péroux, C. 2005, MNRAS, 364, 319

Boissé, P., Le Brun, V., Bergeron, J., \& Deharveng, J.-M. 1998, A\&A, 333,841

Charlton, J. C., Ding, J., Zonak, S. G., et al. 2003, ApJ, 589, 111

Ding, J., Charlton, J. C., Churchill, C. W., \& Palma, C. 2003, ApJ, 590,746

D'Odorico, S., Cristiani, S., Dekker, H., et al. 2000, SPIE, 4005, 121

Ellison, S. L., Yan, L., Hook, I. M., et al. 2001, A\&A, 379, 393

Fall, M., \& Pei, Y. 1993, ApJ, 402, 479

Ferland, G. J. 1997, Hazy, a brief introduction to CLOUDY

Jenkins, E. B., Bowen, D. V., Tripp, T. M., \& Sembach, K. R. 2005, ApJ, 623, 767

Khare, P., Kulkarni, V. P., Lauroesch, J. T., et al. 2004, ApJ, 616, 86

Kulkarni, V. P., Fall, S. M., \& Truran, J. W. 1997, ApJ, 484, L7

Kulkarni, V. P., \& Fall, S. M. 2002, ApJ, 580, 732

Kulkarni, V. P., Fall, S. M., Lauroesch, J. T., et al. 2005, ApJ, 618, 68

Lauroesch, J. T., Truran, J. W., Welty, D. E., \& York, D. G. 1996, PASP, 108, 641

Madau, P., Pozzetti, L., \& Dickinson, M. 1998, ApJ, 498, 106

Malaney, R. A., \& Chaboyer, B. 1996, ApJ, 462, 57

Masiero, J. R., Charlton, J. C., Ding, J., Churchill, C. W., \& Kacprzak, G. 2005, ApJ, 623, 57

Morton, D. C. 2003, ApJS, 149, 205

Nagamine, K., Springel, V., \& Hernquist, L. 2004, MNRAS, 348, 435

Pei, Y. C., Fall, S. M., \& Hauser, M. G. 1999, ApJ, 522, 604

Péroux, C., Petitjean, P., Aracil, B., \& Srianand, A. 2002, New A., 7, 577

Péroux, C., McMahon, R., Storrie-Lombardi, L., \& Irwin, M. 2003a, MNRAS, 346, 1103

Péroux, C., Dessauges-Zavadsky, M., D’Odorico, S., Kim, T. S., \& McMahon, R. 2003b, MNRAS, 345, 480

Pettini, M. 2003, XIII Canary Islands Winter School, ed. Esteban, López, Herrero, \& Sánchez

Pettini, M., King, D. L., Smith, L. J., \& Hunstead, R. W. 1997, ApJ, 478, 536

Pettini, M., Ellison, S. L., Steidel, C. C., \& Bowen, D. V. 1999, ApJ, 510,576

Pettini, M., Ellison, S. L., Steidel, C. C., Shapley, A. E., \& Bowen, D. V. 2000, ApJ, 532, 65

Prochaska, J., Gawiser, E., Wolfe, A. M., Castro, S., \& Djorgovski, S. G. 2003, ApJ, 595, L9

Rao, S. M., Turnshek, D. A., \& Nestor, D. B. 2006, ApJ, 636, 610

Savage, B. D., \& Sembach, K. R. 1991, ApJ, 379, 245

Storrie-Lombardi, L., \& Wolfe, A. 2000, ApJ, 543, 552

Vidal-Madjar, A., Laurent, C., Bonnet, R. M., \& York, D. G. 1977, ApJ, 211, 91

Vladilo, G. 2004, A\&A, 421, 479

Vladilo, G., \& Péroux, C. 2005, A\&A, 444, 461

Welty, D. E., Hobbs, L. M., \& York, D. G. 1991, ApJS, 75, 425

York, D. G., \& the SDSS Collaboration 2000, AJ, 120, 1579

York, D. G., \& the SDSS Collaboration 2001, BAAS, 198, 78.05

York, D. G., et al. 2006, MNRAS, in press

[arXiv:astro-ph/0601279] 